Dr Richard Walker summarises the evidence for a stimulatory effect on haematopoiesis by the products of intestinal bacteria, and it also deals with normal mechanisms for clearing endotoxin after its absorption from the gut.

Even the most dedicated of readers will unfortunately find this book daunting. The series editor has failed to eliminate numerous repetitions and linguistic problems, while the publishers have grossly overpriced the 79 actual text pages of reduced camera-ready typescript. This is a great pity because the subject has important implications for antimicrobial prophylaxis and therapy, as well as for microbial ecology.

SYDNEY SELWYN

\title{
Notes on antimicrobial therapy
}

By Jane Symonds. 1983. Churchill Livingstone, London. Pp. 105. $£ 4.25$.

This small, attractively produced book is designed to serve as a concise guide to antimicrobial treatment for medical students and junior hospital doctors. It largely achieves its aims, and sensibly includes useful short sections on antifungals, antiprotozoals, antivirals and antihelmintics. Errors and criticisms are few, and except for the repeated misspelling of polymyxin are confined to the beta-lactam antibiotics. On the historical side, cephalosporin $\mathrm{C}$ was first reported in 1955, not 1948 - the year of Brotzu's announcement of his initial studies of the Cephalosporium acremomium mould, whose original activities were due to its production of penicillin N. Also, phenoxymethyl penicillin (penicillin V) was not derived from the penicillin nucleus, but is a natural fermentation product when phenoxyacetic acid is used as precursor.

On the more applied side, Dr Symonds' frequent recommendations for the use of mezlocillin in a wide range of infections and of azlocillin in pseudomonas infections should have been accompanied by a note that piperacillin is a convenient single alternative to both drugs. The statement that mecillinam is indicated in "enteric fever and other invasive salmonella infections" would probably not be widely accepted, since the results of such treatment have been equivocal at best. Finally, the erroneous statement is made that "cefazolin" (cephazolin is the correct spelling in the UK) is preferred to cephalexin - and, by inference, cephradine-for the treatment of gram-negative infections "because of enhanced beta-lactamase stability". In fact, cephazolin is the most susceptible of all the currently used cephalosporins to hydrolysis by beta-lactamases; and this defect together with its unusually high degree of protein binding considerably reduce the drug's in-vivo activity. In general, however, this inexpensive little book can be recommended as a sound introduction to antimicrobial therapy.

SYDNEY SELWYN

\section{Microbial skin disease: its epidemiology}

By W. C. Noble. 1983. Edward Arnold Ltd, London. Pp. viii and 135. $£ 15.00$.

In this small book Professor Noble has summarised a considerable body of information on the acquisition of skin infections. After an initial chapter on the microbiology of normal skin, endogenous infections are reviewed. These include such minor disorders as erythrasma, associated with the fluorescent diphtheroid, Corynebacterium minutissimum, and the more complex but analogous disease, acne vulgaris, as well as the true skin infections such as furunculosis, lupus vulgaris and a range of fungal and viral diseases.

It is interesting to note that in subcutaneous abscesses of the head, neck and trunk, propionibacteria are implicated about twice as often as Staphylococcus aureus, while the reverse is the case in the axilla. The only gram-negative skin commensals, Acinetobacter species-the former Mima and Herellea group - are confusingly discussed. Wound and other skin sepsis due to this group is said to occur most frequently in summer, as a result of the promotion of bacterial growth by sweating, yet the accompanying graph shows consistent peaks of infection in winter.

The section dealing with the important group of exogenous infections is apportioned idiosyncratically. Cutaneous diphtheria is given about the same space allocation as streptococ- 
cal infections. The latter are indeed discussed with surprisingly brevity, omitting any mention of infections due to Lancefield groups $\mathrm{C}$ or $\mathrm{G}$. The importance of skin sepsis with specific $\mathrm{M}$ types of group A in the production of acute glomerulonephritis in the tropics is noted in passing, but the paradoxical rarity of rheumatic fever under these circumstances is not touched on.

Viral and fungal infections fare better. Among the less familiar observations is the predilection of dermatophytes for the buttocks and thighs of geriatric patients, and of fungal pathogens of plants for the hands and feet of Indians. The text is concluded with a short section on the modifying effects of the abnormal host and of external environmental factors on skin infections. This nicely rounds off a book that in a modest way will come to be regarded as a standard source of reference.

SydNEY SELWyN 